\title{
Competitive and innovation factors in wine tourism clusters: A comparative study between consolidated and emerging regions in Brazil and Uruguay / Factores de competitividad e innovación en clusters enoturísticos: Un estudio comparativo entre las regiones consolidadas y emergentes en Brasil y Uruguay
}

\author{
Shana Sabbado Flores ${ }^{1, a}$, Claudio Farias ${ }^{2}$, Gabriel Andrade ${ }^{3}$, and Maria Teresa Russi ${ }^{4}$ \\ ${ }^{1}$ Federal Institute of Education, Science and Technology of Rio Grande do Sul (IFRS) - campus Restinga, Rua Alberto Hoffmann \\ 285, CEP 91791-508, Porto Alegre, RS, Brazil \\ 2 Federal Institute of Education, Science and Technology of Rio Grande do Sul (IFRS) - campus Porto Alegre, Rua Coronel Vicente \\ 281, CEP 90300-041, Porto Alegre, RS, Brazil \\ ${ }^{3,4}$ Catholic University of Uruguay (UCU), Av 8 de Octubre 2738, CP. 11600, Montevideo, Uruguay
}

\begin{abstract}
The purpose of this article is to establish a cross-country analysis of the structure and organization of wine tourism clusters in consolidated and emerging wine regions in Brazil and Uruguay, looking for identifying the key factors for competitiveness and innovation. The regions chosen for analysis are: Vale dos Vinhedos, Campanha and Vale do São Francisco, in Brazil, and sites on Montevideo and Canelones, in Uruguay. The study analyze competitive factors in each region, including: the structure and density, support institutions at national and regional level, educational and research institutions, organization process for the geographical indication and the relationship between wine tourism and the promotion of wine and region. Further than comparing the two countries, the research also puts stop regions according to their stage of development in each assessed factor. Thus, the study suggests strategies that can be adopted at regional level or in cooperation between regions (in the country or bi-national cooperation) to strengthen and develop the tourist areas of the wine as a whole.
\end{abstract}

\section{Introducción}

El tema de las estrategias competitivas a pesar de su intenso debate de los años 80, todavía constituye un tema reciente en el campo de la Economía. Por lo tanto, las estrategias innovadoras, el uso de la tecnología y la competitividad son elementos recurrentes, y ampliamente utilizados para entender el desarrollo económico.

Se puede añadir a eso, también, los efectos cada vez más presentes de la globalización (en sus múltiples aspectos y entendimientos), que si por un lado se muestra como una oportunidad, a través de la reducción de las barreras y los límites geográficos entre las empresas, los mercados y países, por otra pone al descubierto las crecientes y aceleradas dinámica del negocio, que requieren las empresas y regiones "hacer mejor y más barato" cada vez más intensa.

Este artículo tiene como objetivo presentar algunas consideraciones sobre las condiciones sistémicas de competitividad y procesos innovadores presentes en la infraestructura turística en las regiones emergentes reciente de la producción de vino en Brasil y Uruguay, el análisis de los impactos de algunas innovaciones

a e-mail: shana.flores@restinga.ifrs.edu.br organizativas en la búsqueda de una mayor competitividad del sector en los últimos veinte años. Cabe destacar que las organizaciones envueltas con el turismo del vino (también conocido como enoturismo) van más allá de las estructuras productivas de uvas y vinos, conformando una especificidad del "rol turístico" - restaurantes, posadas hoteles, agencias de viajes. Tales organizaciones, tanto en las regiones brasileñas cuanto uruguayas, en su extrema maioría, se caracterizan por ser de pequeño porte, prevaleciendo las empresas familiares, y eso ocurre tanto entre productores de uva y vino convencionales, tanto con aquellas empresas más recentemiente asociadas a las actividades turísticas [1].

El objetivo es entender cómo la esfera pública, las asociaciones, las bodegas, agentes y operadores turísticos, restaurantes, artesanías y la hospitalidad se organizan para promover el enoturismo, destacando las diferencias y similitudes, así como los elementos de identificación y componentes de éxito en los modelos adoptados por estas regiones y su impacto en el desarrollo local. El análisis es parte de un proyecto de investigación desarrollado por instituciones de ambos países que buscan un enfoque académico que pueda contribuir al fortalecimiento de las relaciones y el intercambio de información entre las regiones. 
Este trabajo tiene como eje central y de forma exploratoria, lograr tres objetivos: (a) caracterizar, en líneas generales, el sector enoturístico en las dos regiones, en el Brasil y en el Uruguay, su surgimiento, sus instituciones representativas, su dinámica particular en los años; (b) directamente derivado del anterior, verificar los padrones de interacción entre las distintas organizaciones que dan apoyo al desarrollo del sector en cada región; (c) identificar en largos rasgos, los factores de competitividad y de innovación presentes en el sector, en cada una de las regiones.

El artículo se estructura en una primera sección, que abarca una breve discusión sobre la competitividad sistémica y formas e influencias sobre las actividades enoturísticas; una segunda sección que buscará caracterizar el ecosistema que sostiene a las actividades del turismo en regiones productoras de vino en Brasil (en especial en la Serra gaúcha) y en Uruguay (especialmente en la región de Canelones); y una tercera en que se pretende sistematizar las similaridades y diferencias, mostrando algunas consideraciones en relación a la estructura institucional que da la sustentación al enoturismo en las regiones analizadas. Por lo tanto, se llevó a cabo entrevistas con expertos de la industria, y con una revisión de la literatura y datos estadísticos recientes.

\section{Competitividad sistémica $Y$ enoturismo}

Aunque frecuente, los estudios de competitividad presentan disonancias conceptuales en las metodologías empleadas en estas evaluaciones. Sob el punto de vista interno a la empresa, la competitividad presenta una diversidad de conceptos. Para efectos de las análisis presentadas en este trabajo, se llevó en cuenta el concepto utilizado en el Estudio de la Competitividad de la Industria Brasileña (ECIB), que presenta la competitividad como siendo "la capacidad de la empresa de formular y implementar estrategias competitivas, que les permitan ampliar o conservar, de manera durable, una posición sustentable en el mercado" [2]. Ese concepto introduz el carácter dinámico en el análisis de la competitividad, entendiendo por competitiva la empresa que modifica continuamente la propia estructura, la adaptación a una mayor o menor medida, el contexto del mercado en el que está instalado o piensa instalar.

El término competitividad no posee un concepto ampliamente empleado, sea en la academia, sea en los medios empresariales o gobernamentales. Se utiliza la palabra competitividad, de manera amplia y general, para expresar la comparación, la competencia o benefícios. Por último, la competitividad expresa un conjunto de factores de gestión, la innovación, la producción y los recursos humanos que pertenecen a la misma realidad dinámica. La dificultad de definir un concepto único de competitividad es mencionada por Barbosa [3], que dice que diferentes autores hacen en diferentes aspectos $\mathrm{y}$ en ocasiones con la competitividad conceptos totalmente singulares y únicos. También se dice que el problema es decidir qué aspectos cualitativos y cuantitativos deben ser destacados. Comenta, también, que el problema es decidir cuáles aspectos cualitativos y cuantitativos deberían ser enfatizados. Para algunos autores, competitividad es una cuestión de aumentar el índice de vida de una nación; para otros, el concepto está arraigado con la habilidad de una empresa en competir y negociar con éxito sus productos y servicios en escala mundial.

El éxito de la competitividad pasa a depender de un conjunto de factores que influencian el desempeño de las empresas. De acuerdo con Coutinho e Ferraz [2], la noción de competitividad sistémica parece adecuada como modo de expresar que el desempeño empresarial depende y es también resultado de los factores externos al ámbito de las empresas y de la estructura industrial; depende de los factores sistémicos relacionados a las políticas macroeconómicas adoptadas por un país, de los factores estructurales relativos a las políticas sectoriales y de los factores internos de cada empresa, denominados factores empresariales, relacionados a las prácticas adoptadas y controladas por la empresa.

Según Lapolli et al. [4], combinación interactiva de varios factores que actúan en el sentido de que la competitividad sea su resultado. Entre los factores determinantes de la competitividad, Lapolli, et al. destacan:

(a) Factores internos el precio, a su vez, debido a los costos; la calidad, entendida en un sentido ajustado a un mercado en particular con su venta y consumo normal correspondiente; la productividad; la tecnología; estrategia competitiva, buscando las condiciones de la eficiencia global de la empresa y lo que implica decisiones en varias áreas.

(b) Factores Externos: ambiente macroeconómico, que consta de varios componentes, tales como la tasa de inversión, el mercado de trabajo, la productividad, el equilibrio de la balanza comercial, la disponibilidad $\mathrm{y}$ el costo de los fondos, las organizaciones y las empresas a la cooperación empresarial, la organización y las relaciones laborales, el nivel de salario y calificación de los recursos humanos; infraestructura económica; infraestructura técnicocientífica y educacional; estructura sectorial; uso de instrumentos de política económica y dimensión institucional. Para Coutinho y Ferraz [2], el desarrollo competitivo de una empresa, industria o de una nación depende de una amplia gama de factores internos de la empresa, la estructura (sectorial) y de la naturaleza sistémica.

El enfoque sugerido por Ferraz et al. [5], se enfatiza como determinantes de la competitividad de un número de factores, además de ser en gran número, trascender el nivel de la empresa, y también están relacionados con la estructura de la industria y del mercado y también del sistema de productivo en su conjunto como un todo. Los factores determinantes de la competitividad, de acuerdo con Ferraz et al....[5], fueron definidos en tres grandes grupos: factores empresariales, los factores estructurales y factores sistémicos.

Los factores empresariales, como muestra Ferraz et al. [5] son aquellos que, perteneciendo a la empresa, sobre los cuales la empresa detiene el poder de decisión y pueden ser controlados o modificados mediante un comportamiento activo asumido, lo que corresponde a las variables en el proceso de tomada de decisiones. Estos se refieren a las cuatro áreas de competencia de la empresa: Gestión, Innovación, Producción y Recursos Humanos. 
Los factores estructurales, según los mismos autores, son aquellos en los que la capacidad de la empresa para intervenir se limita midiendo el proceso de concurrencia y, por tanto, sólo son parcialmente bajo su área de influencia. Estos se refieren tanto a la organización de la producción intra-sectorial y las relaciones entre proveedores y productores en las cadenas productivas.

Los factores sistémicos a Ferraz et al. [5] son aquellos que constituyen externalidades, estrictamente hablando, para la empresa productiva sobre las que la compañía tiene poco o ningún poder para intervenir, constituyendo parámetros de la toma de decisiones. Estos factores pueden determinar ventajas competitivas para las empresas en relación con los competidores de otros países. De este modo, se pueden agrupar: macroeconómicos, político-institucionales, legales-regulatorios, infraestructurales, sociales y internacionales.

Uno de los modelos analíticos más ampliamente utilizados es el modelo de "diamante", por Michael Porter. El modelo tiene como objetivo presentar un marco analítico, de las zonas (clusters) con especial desarrollo económico.

\subsection{MODELO DE PORTER: la idea de cluster y los factores determinantes de la competitividad}

A pesar del concepto de cluster no ser exclusivo de Porter, es inevitable reconocer que se le da un significado particular al concepto. Porter [6] trata el término como un agrupamiento de industria competitivas, entendidas como un éxito, ya que por lo general se conectan a través de verticales (comprador / proveedor) u horizontales (clientes, tecnologías, canales comunes, etc.).

El autor señala que este agrupamiento deriva de la naturaleza sistémica de lo que llamó "diamante", una expresión utilizada para referirse a los factores determinantes de la ventaja nacional como un sistema. Así que para Porter [6], las empresas de un país tendrán éxito si los cuatro atributos principales que dan forma al ambiente en el que compiten las empresas y promover la creación de ventajas competitivas, a saber: 1) Condiciones de factores (tales como mano de obra especializada y la infraestructura necesaria para la competencia); 2) las condiciones de demanda (la naturaleza de la demanda interna de productos); 3) las industrias y el apoyo (la presencia de apoyo a las industrias que son competitivos a nivel internacional) relacionados; 4) Estrategia, estructura y rivalidad de las empresas (las condiciones que rigen la manera en que se crean las empresas, organizadas y dirigidas). Además de los cuatro atributos enumerados anteriormente, Porter [6] identifica dos variables, el azar y el gobierno, como una contribución a la formación de la mayoría de las industrias exitosas.

El acaso son ocurrencias aleatorias, que tiene poco que ver con las circunstancias de un país o región, y están fuera del alcance de las empresas, ya menudo demasiado lejos de los gobiernos nacionales. Porter [6] cita los siguientes ejemplos: los actos de pura invención; principales discontinuidades tecnológicas (como la biotecnología y la microelectrónica); discontinuidades en los costos de las materias primas (por ejemplo, los choques del petróleo); cambios significativos en los mercados financieros internacionales y tipos de cambio; los aumentos repentinos de la demanda mundial o regional; las decisiones políticas de los gobiernos extranjeros; guerras.

El papel de los gobiernos, o de las políticas del gobierno, el autor confiesa la tentación de adoptarlo como un factor determinante en sí, dada su importancia en la construcción de la competitividad de las industrias nacionales. Sin embargo, Porter [6] admite que el verdadero papel del gobierno en la ventaja competitiva es influir en los cuatro diamantes y al mismo tiempo ser influenciado por ellos. Por lo tanto, Porter [6] identifica los principales potenciales interacciones entre los gobiernos y los cuatro diamantes, que son:

a) las condiciones de los factores se ven afectados por las subvenciones, las políticas hacia los mercados de capitales, etc. políticas educativas;

b) modelar la demanda de las condiciones locales;

c) las normas de los establecimientos o la normativa local sobre productos, que afectan o influyen en las necesidades de los compradores;

d) el gobierno es también, en muchos segmentos, un fuerte comprador de muchos productos;

e) el gobierno puede dar forma a las circunstancias de las industrias relacionadas y de apoyo de muchas otras maneras, como el control de los medios de publicidad y regulación de los servicios de apoyo;

f) Las políticas del gobierno pueden influir en las estrategias de negocio y la rivalidad, a través de características tales como la regulación de los mercados de capitales, la política fiscal y las leyes antimonopolio;

g) la política del gobierno puede ser, a su vez, la influencia de los factores determinantes. Las determinaciones de las inversiones en políticas educativas específicas, una mayor regulación y subsidios son ejemplos comunes de formas de influir en las políticas gubernamentales, llevadas a cabo por empresas o sus representaciones.

Por lo tanto, a partir de una lectura preliminar del autor, uno puede entender los clusters como concentraciones geográficas de compañías e instituciones interconectadas en un área de especialización. Los clusters incluyen proveedores especializados de insumos tales como componentes, maquinaria, servicios y proveedores de infraestructura. Los racimos se extienden la cadena de producción para incluir canales y fabricantes de productos complementarios de marketing, llegando a empresas relacionadas por la cualificación de los recursos humanos, tecnologías o insumos locales. Por último, las agrupaciones incluyen gubernamentales y de otro tipo, tales como universidades, instituciones de control de calidad, centros de investigación, y las asociaciones de empleadores y los sindicatos, que ofrecen una serie de servicios técnicos especializados para la industria.

Porter [7] cita como un ejemplo exitoso de clúster el caso de la industria del vino de California. La idea de cluster desborda la simple relación entre viticultores y la industria del vino. Esta relación se consolida geográficamente, incluidos los organismos gubernamentales (responsables de la formulación de políticas públicas) y educativas y centros de investigación, así como el tráfico de órganos. Al mismo tiempo, una serie de segmentos industriales se juntan alrededor 
de la prestación de servicios y entradas de marketing a los productores de uva; También, un centenar de empresas ofrecen servicios especializados e insumos para la producción de vinos.

Cabe, por lo tanto, comprender y analizar la estructura del turismo del vino en Brasil y Uruguay, desde la concepción de los factores sistémicos que hacen parte de que cada una de las regiones. Para el análisis de este estudio, empleamos el concepto de determinantes sistémicos de la competitividad del turismo del vino de las regiones analizadas, Brasil y Uruguay. Se señala que se va a utilizar el modelo de Porter, para entender cómo las políticas y acciones de apoyo han actuado como factores determinantes del turismo del vino en ambas regiones.

\subsection{Enoturismo}

La idea del enoturismo está en la práctica de las actividades de ocio relacionadas con el mundo del vino. Hall et al. [8] explican que el enoturismo puede estar motivado por la región de vino en sí, por las actividades relacionadas con el vino (como degustaciones, por ejemplo), o en ambos casos. Al mismo tiempo, el concepto de turismo incluye una serie de situaciones que puede implicar, por ejemplo, actividades recreativas, excursiones y/o visitas.

Técnicamente hablando, la actividad sería una variación o contextualización, de prácticas muy presentes en la economía. Sin embargo, el enoturismo es considerado especial y diferenciado, ya que ofrece un conjunto de atributos estéticos y regionales [8]. Sommers [9] señala que pocas actividades poseen una relación tan estrecha entre el pueblo y el cultivo como la vitivinicultura; en consecuencia, este tipo de turismo acaba por traer a los visitantes, ideales rurales que os conecta con la tierra y con la gente local. La actividad no está vinculada a una herencia de turismo industrial, pero se refiere a los aspectos históricos y culturales. En la medida en que ponen de relieve el vínculo con la naturaleza, las actividades relacionadas con el enoturismo animan a los visitantes a sumergirse y quieren aprender más sobre el mundo del vino [9].

El enoturismo acaba por unir dos industrias, las dos ambas con implicación sustancial en el territorio [8,9]. Del punto de vista de las vinícolas y de los productores de vino, las principales vantajes experimentadas provienen de una mayor proximidad con el cliente. Así que, en la medida en que los visitantes pasan a tener un mayor contacto $\mathrm{y}$ conocimiento de los productos, la percepción y fidelidad pueden mejorar, posibilitan por su vez, también, vínculos subjetivos entre producto y consumidor. Ese contacto directo es una oportunidad de comercialización, sobretodo para los productores de pequeño porte, que tendrían mayor dificultad de inserción de venta al por menor, además de la eliminación de los intermediarios, lo que aumenta los márgenes. Por otra parte, la iniciativa trae aumento de los costos y complejidad en las operaciones, ya que requiere un nuevo perfil de acción, que se diferencia de la industria del vino puro y simple, que requiere nuevas habilidades. En este sentido, es de destacar la inversión en una estructura específica para atender a los turistas [8].

En un panorama internacional, se observa que el enoturismo es un pilar importante en los territorios de los vinos del "Nuevo Mundo". Para tener una idea, se estima que las actividades de visita y turismo del vino en general están presentes en el $90 \%$ de las bodegas en Australia y el $60 \%$ en Nueva Zelanda. Napa Valley (California / EE.UU.), por ejemplo, uno de los principales exponentes de la zona, recibe alrededor de 5 millones de visitantes al año, que manejan 600 millones de dólares (Cambourne et al., 2000). En Brasil, el área principal es el Vale dos Vinhedos, que recibió 283.000 visitantes en 2013. A partir del proceso de la Indicación Geográfica, que mejora el desarrollo de la región del vino en su conjunto y su organización para el turismo del vino, la zona ha sufrido una serie de transformaciones.

La intensificación de la actividad tornó el Vale dos Vinhedos en la región con la mayor concentración de vinícolas del país, con 21 establecimientos, distribuido unos 30 de ellos $81 \mathrm{~km}^{2}$ de la región demarcada [10]. Los impactos del enoturismo en el caso del Vale está más allá de la escala económica, tocando aspectos sociales y ambientales, tales como la mejora de la producción y la propiedad o la aparición y el desarrollo de las distintas actividades productivas y comerciales, la generación de empleos e ingresos. En el plano de la infraestructura, se destaca la pavimentación de caminos rurales, la instalación de casas de huéspedes y hoteles, el crecimiento del consumo de electricidad y teléfono, además de la modernización de las condiciones de vivienda. Todo esto también genera una mayor carga de residuos domésticos e industriales, el aumento del tráfico de automóviles y autobuses, y la pérdida de diversidad de especies de plantas [11].

\section{Enoturismo en Brasil y en Uruguay: factores competitivos e innovaciones}

Para analizar las regiones vitivinícolas del Brasil y de Uruguay, fueron elegidos 5 factores fomentadores de la competitividad y la innovación: (1) la estructura y densidad, (2) instituciones de apoyo a nivel nacional y regional, (3) instituciones educativas, (4) procesos de organización de investigación para la indicación geográfica y (5) la relación entre el turismo y promoción del vino y la región. En la estructura del artículo y la densidad se observa la estructura de la cadena en general, incluidos los principales productores de concentración de bodegas y viñedos, así como la presencia de la estructura de soporte del enoturismo, tales como: agencias, bares y restaurantes, hoteles, así rutas organizadas. Un punto importante en este sentido es la densidad en términos de las atracciones turísticas y enoturísticos, que pueden contribuir directamente a la promoción de las rutas.

Con relación a las instituciones de apoyo, se separaron en dos factores que deben ser analizados, el primero en las instituciones de investigación y apoyo en general, incluidas las asociaciones locales para el turismo y la promoción de la región vinícola, los organismos nacionales para la promoción de la industria del vino como un todo. Además, se hizo hincapié en las instituciones educativas, la comprensión que contribuyen directamente a la formación de profesionales y trabajan con la investigación en las regiones y temas de interés. Por otra parte, la presencia de instituciones de educación superior se relaciona con los procesos de innovación y empreendedorismo en una serie de contexto de la investigación internacional, tales como el Informe GEM (Global Monitor de Entrepreneuship). 
Ya las indicaciones geográficas (IG) corresponden a una temática intrínsecamente relacionada con el mundo del vino. El proceso termina por formalizar la región del vino, el reconocimiento de los actores involucrados, y el establecimiento de normas de calidad de los productos. Aunque en su origen no estar directamente relacionado con experiencias del enoturismo, como el Vale dos Vinhedos muestran que el proceso contribuye a la proyección de la región, con el potencial de estimular a otros segmentos unidos a la cadena de vinos, entre los cuales, se destaca el enoturismo. Por último, se destaca como un factor de la relación entre el enoturismo y la promoción del turismo en la región en su conjunto. En este sentido, el objetivo fue evaluar la relación entre el turismo relacionado al mundo de los vinos y otras iniciativas relacionadas con la región, la organización sectorial y si el turismo relacionado con los vinos serían la atracción principal o actuaría de manera complementaria en la realidad local. Por lo tanto, se hará un análisis de la sección transversal de cada factor para las regiones seleccionadas.

\subsection{Estructura y densidad}

La estructura del artículo y la densidad se evaluó en dos aspectos principales: estructura y densidad de los viñedos, la concentración de la producción y el enoturismo. Considerando que el enoturismo trae la unión de dos industrias, la producción de vino y del turismo, se consideró que el análisis del enoturismo debería considerar de la producción de vino, junto con las estructuras para el turismo en sí.

Los viñedos brasileños hoy ocupan un área de 83,7 mil hectares, de los cuales $60 \%$ en vitis vinífera [12]. La provincia del Rio Grande do Sul es el principal productor nacional, con safra anual entre 600 e 700 millones de toneladas, es responsable por el cultivo de las uvas de $90 \%$ de la producción nacional de vinos y jugos (Ibravin, 2013a; b). En la Serra Gaúcha, principal región productora, se destaca el Vale dos Vinhedos (en los departamentos de Bento Gonçalves, Garibaldi, Monte Belo do Sul), con aproximadamente $81 \mathrm{Km}^{2}$ son 8 emprendimientos entre hoteles y posadas, 20 restaurantes y 24 vinícolas. La Serra Gaúcha fue el escenario de la real consolidación de la vitivinicultura en Brasil, que empezó con la llegada de los inmigrantes en el siglo XIX, en especial los alemanes, más tarde, italianos, a eso de 1875, responsables por el grande impulso de la vitivinicultura en el país [13].

Los viñedos emergentes tratados aquí se hicieron sobre una base diferente y son parte de la última expansión en el sur y noreste del país, precisamente, a partir de la década de 1980, en una convergencia de la inversión extranjera, las iniciativas locales e inversiones de las bodegas de la Serra Gaúcha, que amplió sus áreas de producción en las regiones de la Campaña (RS) y el Valle de San Francisco. Hoy en día, ambas regiones tienen viñedos, bodegas, asociaciones industriales, instituciones educativas y de investigación en el área, las iniciativas de turismo del vino, así como los proyectos en curso para la indicación geográfica, en un movimiento de consolidación, que se intensificó a partir de 2010. La Campaña cuenta con 11 departamentos con un total, de casi 100 productores y cerca de 1600ha; 7 departamentos poseen alguna propuesta en términos de enoturismo. Los principales productores son: Santana do Livramento, Candiota, Bagé y Dom Pedrito.
A su vez, el Valle de São Francisco, una región situada entre las provincias de Pernambuco y Bahía, es la principal región tropical de vino de Brasil, con cerca de 11 hectáreas de viñedos [14]. Alrededor del 95\% del área plantada de viñedos es para la producción de uva de mesa, destinada principalmente al mercado de exportación. La región representa casi la totalidad de la producción de uva de mesa de exportación. En cuanto a los vinos, la región es el principal exponente de la viticultura tropical, con el diferencial de producir hasta cinco cosechas a cada dos años. La región ha llegado a producir 7 millones de litros por año, ahora tiene cerca de 360 ha y cinco bodegas. La producción de vino ha aparecido en el Estanque Grande, con tres bodegas y el volumen más grande de la región, que se distribuye en los vinos tintos, blancos y espumosos. Casa Nova ya concentra el $80 \%$ de su producción en el brillante, especialmente la variedad moscatel. También se destaca la preparación de brandy. Los dos departamentos tienen las iniciativas del eno turismo.

En Uruguay, la región de Canelones concentra la mayor parte del cultivo vitícola con el $67 \%$ de los viñedos, que ocupan el $64 \%$ de la superficie del departamento y el $74 \%$ de la producción de uva con respecto al total nacional. Cuenta con cerca de 150 establecimientos de los cuales solo unos pocos se dedican al enoturismo. Montevideo es el segundo productor vitivinícola del país, contando con el $14,9 \%$ de los viñedos, que ocupan el $12,5 \%$ de la superficie del departamento y el 13,2\% de la producción de uva con respecto al total nacional. Cuenta con cerca de 90 establecimientos de los cuales, al igual que en Canelones, solo unos pocos se dedican a la actividad del enoturismo.

Con relación a la representatividad del vino en el territorio, es posible percibir que la viticultura tiene un papel más central en el Vale dos Vinhedos (y Serra Gaúcha) y en Canelones. Las regiones poseen en común el facto de que representan la mayor parte de la producción vitivinícola nacional, con una buena densidad de viñedos y establecimientos vitivinícolas. En el caso de Montevideo, aunque tenga una representación más pequeña de que Canelones hacia el país, la región tiene una buena estructura y densidad de propiedades. En los territorios emergentes de Brasil, es posible ver que la propia viticultura es una actividad más reciente y no predominante, incluso en el contexto del territorio. En el caso de VSF, uvas de mesa tiene un papel importante en la economía local y representa casi la totalidad de la producción nacional para exportación, pero la producción de vinos tiene una escala bien menos representativa.

Otro punto a considerar con respecto a la estructura y la densidad es el tamaño de las propiedades y la concentración de la producción. Cabe señalar que en algunas regiones la producción tiende a ser más distribuida en más puntos de venta, con concentraciones más bajas de producción y superficie cultivada. En el siguiente diagrama (Fig. 1) se puede observar con respecto a la concentración de la producción y del área del cultivo. Mientras que la producción en el Vale dos vinhedos, Canelones y Montevideo están distribuidos en el territorio en varios establecimientos en el otro extremo que es el Valle del São Francisco, con la producción concentrada en cinco bodegas. Este aspecto se refiere al tamaño medio de las propiedades, sino que también indica la presencia de la agricultura o la empresa familiar en la zona. La Campaña estaría en una posición intermediaria. Este análisis no 


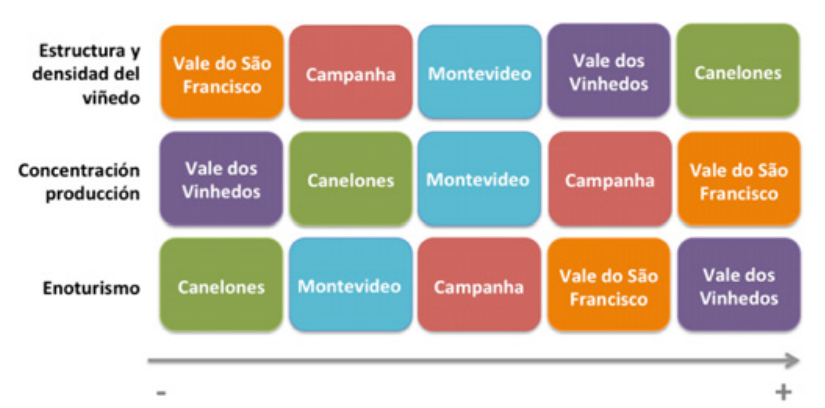

Figura 1. Escala de las regiones con relación a los criterios de estructura y densidad. Fuente: los autores.

quiere decir que los territorios son uniformes: por supuesto que existen los establecimientos más grandes en el Vale dos vinhedos y de las regiones del Uruguay. Sin embargo, la densidad de la mayoría de los establecimientos también indica una mayor distribución de la producción en el territorio.

En cuanto a la estructura y densidad al turismo del vino, las regiones de Brasil son más estructurados, con iniciativas a nivel de la región vinícola de promover excursiones o vino de forma individual. Incluso en las regiones con menor densidad en términos de producción, o una concentración más alta, se puede observar en términos relativos, el enoturismo es una actividad importante para las bodegas de la zona y la mayoría de ellos tienden a ofrecer propuestas concretas o integrarse en itinerarios con otros segmentos. En Uruguay, está claro que las bodegas sólo se han centrado en la producción y la exportación, mientras que en Brasil el enoturismo es una herramienta importante no sólo para la promoción del vino, pero termina por convertirse en una importante fuente de receta relevante. Cabe señalar que Uruguay gasta un esfuerzo importante en la industria del turismo en su conjunto, y en este caso, tiene la más larga tradición y la condición de la competencia, especialmente en el análisis de la "captura" de turistas internacionales, en comparación con Brasil. Por lo tanto el enoturismo es otra variante del "rol" turístico del Uruguay.

\subsection{Instituciones de apoyo a nivel nacional y regional}

Las instituciones de apoyo son un pilar importante para impulsar la competitividad de la región vinícola. En apoyo de las instituciones se consideran las instituciones gubernamentales o de la industria del turismo de soporte del vino, asociaciones profesionales o industriales, y los institutos de investigación y extensión. En Brasil, las instituciones de apoyo para la uva y el vino se concentran en la Serra Gaúcha. Hay un apoyo directo a las instituciones y el turismo del vino indirecta en la región de los cuales destacamos el Instituto Brasileño del Vino (IBRAVIN), la Unión Brasileña de Vitivinicultura (Uvibra) y Embrapa Uva y el Vino en el plano nacional. Aún así, se destaca la Innovación Red Centros en Viticultura, promovido por IBRAVIN y vinculada al Sistema de Tecnología de Brasil (SIBRATEC), el Ministerio de Ciencia y Tecnología, que opera con fondos de la FINEP (MCT, 2010). Para Serra Gaucha soportar la Asociación Gaucha de Enólogos (AGAVI) y la Asociación de Viticultores de Garibaldi (AVIGA), mientras que la

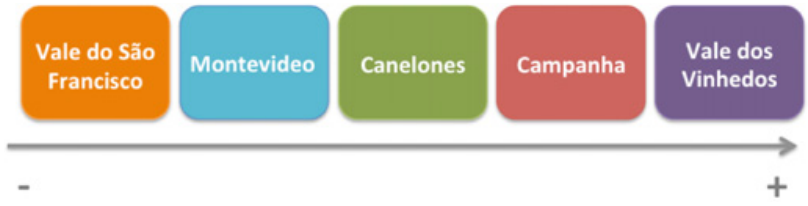

Figura 2. Escala de las regiones con relación a los criterios de instituciones de apoyo. Fuente: los autores.

Asociación de Bellas Productores de Vino del Valle de los Viñedos (Aprovale) es la principal fuente de apoyo en el Vale dos Vinhedos que tiene papel clave en la región, su organización y en el proceso de la indicación geográfica [15].

Con relación a la Campaña, para reunir y representar a las bodegas de la zona, el territorio tiene la Asociación "Campaña Vino", que ha desempeñado un papel importante en la promoción de acciones en la región, más allá del proceso de la Indicación Geográfica. Se formó la Asociación inspirada en el modelo de la Aprovale y reúne a las bodegas locales y asociaciones de productores de vino. Además, la región cuenta con asociaciones locales se centraron en la viticultura o la fruta (que incluyen la presencia de viticultores), las principales asociaciones son la Asociación de productores de Santana do Livramento uva (ASPROUVA) Asociación de Productores de Frutas Quariense (AQUAFRUTI) Asociación Bageense de la fruta (ABEFRUT) y la Asociación de fruta de Uruguaiana (AFRUG). El Vale do São Francisco cuenta con una unidad de la Embrapa Uva y Vino, vinculada localmente con la Embrapa Semiárido, para el desarrollo de pesquisas y apoyo técnico. Para reunir y representar las bodegas locales, el territorio posee el Instituto del Vino del Vale do São Francisco (VinhoVASF).

En Uruguay se cuenta con un importante apoyo del Instituto Nacional de Vitivinicultura (INAVI), la Asociación Los Caminos del Vino, Uruguay XXI en promoción de exportaciones de bienes y servicios, las Intendencias Departamentales y del Ministerio de Turismo del Uruguay. Dadas las características geográficas de Montevideo y Canelones, en general el tema del vino se trabaja en conjunto, las Intendencias departamentales tienen un fuerte rol local, pero las asociaciones privadas incluyen establecimientos de los dos departamentos.

\subsection{Instituciones educativas}

En el tema de las instituciones de educación se resaltan las instituciones que tienen influencia en las regiones encuestadas, actúan localmente en la formación profesional o de investigación en el área, incluso sin cuartel en las regiones.

En Serra Gaúcha, las principales instituciones son la Universidad de Caxias do Sul (UCS), el Instituto Federal de Educación, Ciencia y Rio Grande do Sul Tecnología (IFRS) y la Universidad Federal de Rio Grande do Sul (UFRGS). La UCS tiene su historia ligada al desarrollo de la industria del vino de la tierra, con una actividad significativa desde el punto de vista de la investigación y la extensión. El UCS contribuyó directamente en la investigación que llevó a la IG Vale dos vinhedos, y apoyar el reconocimiento de otros soldados en la Serra Gaúcha (por ejemplo, Pinto Bandeira, Monte Belo y Farroupilha); 
hoy la universidad tiene una maestría en biotecnología centrada en los estudios de la uva y del vino. IFRS fue la primera institución brasileña a ofrecer cursos de enología y viticultura en los años 60, en la vieja Escuela Agrotécnica y en la actualidad es el campus de Bento Gonçalves del IFRS; actualmente la institución ofrece una formación técnica, superior y especialización; un título de maestría profesional en el campo está para comenzar en 2017. La UFRGS no tiene la sede en la Sierra, está en curso la implementación de un campus avanzado; sin embargo, la universidad está trabajando en la investigación relacionada con la uva y el vino en la provincia en su conjunto y en particular en la región, especialmente en el nivel de posgrado.

En la Campaña, que pone de relieve la Universidad Federal del Pampa (UNIPAMPA) que tiene la primera y única Licenciatura de Enología del país, en el campus de Dom Pedrito. Además, el campus Pelotas del Instituto Federal de Rio Grande del Sur (IFSul) ofrece el curso de Viticultura y Enología tecnólogo. Se destacan en las inversiones federales, que la región recibió en los últimos 10 años. La región también tiene Polos de Educación a Distancia (EAD). Este movimiento dio lugar a la calificación en el nivel superior y técnica, en varias áreas, incluyendo la industria del vino. Otras instituciones involucradas en la investigación en la región son la Universidad Federal de Santa María (UFSM) y la Universidad Federal de Pelotas (UFPel), que también contribuyen a la formación de profesionales en el área de nivel de posgrado.

En el Valle del São Francisco, el Instituto Federal de Pernambuco (IFSertãoPE) alberga la "Escuela del Vino" y proporciona formación en el área del tecnólogo en Enología y Viticultura incluso los cursos de corta duración, como Operador de la producción Vitinícola y, más recientemente, Sommelier. Junto a esto, el campus de la Universidad de Juazeiro Estadual de Bahía (UNEB) tiene curso de Agronomía, especializaciones y maestrías, lo que abre la posibilidad de la asignatura en el ámbito de la fruticultura y vitivinicultura de irrigación.

En Uruguay existe una oferta de formación técnica que aporta cursos de corta duración. La formación técnica depende de la facultad de Química con un curso de profissional de Sommelier y por otro lado, la Escuela Superior de Vitivinicultura "Pte. Tomás Berreta", perteneciente a Universidad del Trabajo del Uruguay (UTU), en Canelones, ha sido la responsable de la formación de los Enólogos - su fundación data del año 1939 y es la precursora de las buenas prácticas vitivinícolas. A nivel Universitario la Universidad Católica del Uruguay (UCU) cuenta con la Licenciatura en Dirección de Empresas Turísticas. Ninguna propuesta aún está especializada en el tema vitivinícola. La Escuela de Enología y la Facultad de Química son las únicas propuestas técnicas vinculadas al sector.

Para hacer el análisis comparativo de las regiones estudiadas, se utilizó como criterio la presencia y densidad de las instituciones educativas que ofrecen cursos para apoyar el turismo del vino, así como su integración con el arreglo productivo local. La escala puede verse a continuación en la Figura 3. Se puede observar que las regiones brasileñas tienen un mayor vínculo con la disposición de turismo del vino, que se produce tanto en la organización de cursos y en propuestas concretas

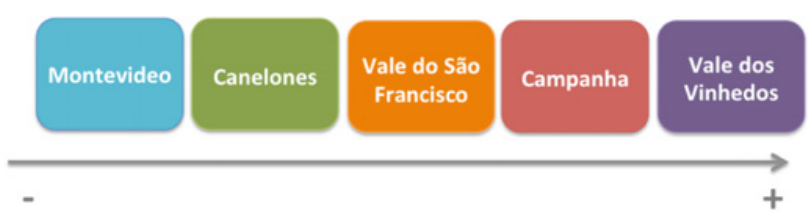

Figura 3. Escala de las regiones con relación a los criterios de instituciones de educación. Fuente: los autores.

de turismo del vino o enogastronomia. El Vale dos vinhedos tiene una serie de instituciones de enseñanza y de investigación que actúan de manera decisiva en los procesos importantes de mejora técnica y la estructuración de las regiones, como indicaciones geográficas. Incluso en las regiones emergentes de Brasil, que dependen de las instituciones para menores, ya se puede ver una preocupación con el enoturismo y la participación en este proceso. Por otro lado, las instituciones uruguayas tienen una tradición con formación técnica en el área de cultivo de la viña, lo que contribuye de manera significativa al desarrollo de la viticultura en la región. Al mismo tiempo, hay una formación en turismo y gastronomía; pero este tipo de formaciones terminan interactuando menos con el enoturismo, que se dirige más a otras áreas.

\subsection{Indicación geográfica}

El proceso de la indicación geográfica es muy importante en el contexto de Brasil, ya que además de reconocer y proyectar la región, que en última instancia contribuye a una mayor articulación de los actores, que tiene repercusiones en otras esferas. En este sentido, los vinos también han desempeñado un papel central en el desarrollo de las indicaciones geográficas en Brasil, fueron los precursores de este proceso y siguen siendo una referencia para las indicaciones geográficas en otras áreas.

En el vale dos vinhedos, el proceso de IG se inició en 1995 por iniciativa de los productores locales, con la creación de la Asociación de Bellas Productores de Vino del Valle de viñedos (Aprovale). La indicación de procedencia para la región do vale dos vinhedos (IPVV) se delimitó en 1999 y se estableció en noviembre de 2002, la primera IG Brasil (Falcade 2007). A partir de 2012 IG Denominación de Origen (DO) que implica el nivel más específico y más alto de calidad para la producción de vinos y espumantes.

En la Campaña, la indicación geográfica proceso está ligado a la Red de Centros de Innovación en Viticultura, siendo coordinado por la Embrapa Uva y el Vino y conectado a SIBRATEC, el Ministerio de Ciencia y Tecnología, con fondos de la FINEP. La investigación se inició oficialmente en 2013, y la previsión es de reconocimiento en 2016. El proceso de IG ha contribuido a una mayor coordinación de los actores y jugó un papel decisivo en la formación de la Campaña de la Asociación de vino. En el Valle del São Francisco el proceso de IG ha estado en curso desde 2015, coordinado por Embrapa Uva y el Vino y con una estructura similar a los procesos de IG que tienen lugar en la Serra Gaúcha. Se pone en destaque que la Campaña y el Vale do São Francisco son las únicas áreas fuera de la Serra Gaúcha que iniciaron el proceso de indicación geográfica. 


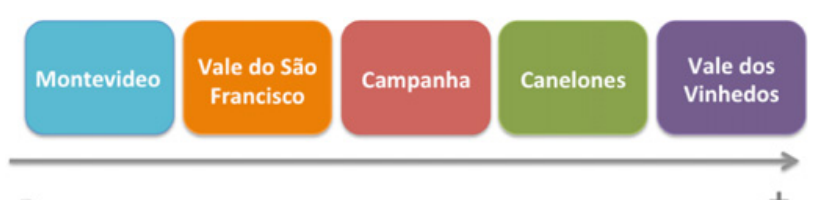

Figura 4. Escala de las regiones con relación a la Indicación Geográfica. Fuente: los autores.

En Uruguay existe una iniciativa del Ministerio de Turismo del Uruguay para generar referencias geográficas de establecimientos productivos y atractivos turísticos en general. Para los vinos, segundo el INAVI, la región de Canelones cuenta con tres IGs: Juanicó, desde 1994, Canelón Chico y Atlántida, desde 2002. No hay todavía una articulación entre la indicación geográfica de la región vinícola y los actores para la promoción del enoturismo.

La Figura 4 muestra un diagrama con la escala de desarrollo en lo que respecta a la indicación geográfica. En este sentido, es importante que los países tienen diferentes marcos legales, y diferentes procesos para el reconocimiento de las indicaciones geográficas, por lo que es difícil establecer una escala de comparación. Para establecer la escala se consideró la existencia de las indicaciones geográficas y la profundización en el primer lugar. Por lo tanto, el Vale dos vinhedos aparece en primer lugar porque, a pesar de haber reconocido por primera vez después de IG Canelones, el proceso se ha convertido en la categoría de origen. Al mismo tiempo, el caso del Vale dos vinhedos que contribuye directamente sólo a la articulación de la región y la promoción del turismo del vino. Luego vinieron las regiones brasileñas que ya tienen GI en curso, seguido de Montevideo.

\subsection{Turismo y promoción del vino y de la región}

En ese tópico se evalúa la relación entre el vino y el enoturismo y la promoción de las regiones. En la Serra Gaúcha está aumentando la participación del turismo en la promoción del vino de la tierra. Fuerte tendencia de las bodegas para adoptar los proyectos de turismo programas / vino. El turismo del vino también ha sido una estrategia importante para las pequeñas bodegas que producen vinos y zumos de uva coloniales, la diversificación de las actividades rurales, con el fin de ampliar los ingresos familiares y la apreciación del producto regional. Al mismo tiempo, el vino, históricamente ha sido utilizado para promover la región de Serra Gaucha y su industria; por citar un ejemplo, la historia de la Fiesta de la Uva en Caxias do Sul está relacionado principalmente con la idea de promover la industria metalúrgica y metal-mecánica en la región, y no de sus uvas y vinos [10].

Tras la tendencia de inversión en enoturismo, las bodegas de la Campaña han estado trabajando para establecer rutas regionales, combinando las propuestas con elementos de la cultura gauchesca o con compras en el turismo fronterizo. El enoturismo es un importante canal para la venta directa de vino. Una curiosidad es que algunas bodegas han invertido en enoturismo (o planes de inversión) en otras regiones turísticas de Brasil, como la Serra Gaúcha o incluso el estado de São Paulo. Otro punto es la proximidad con Uruguay, al igual que en el caso de la festividad Bi-Nacional de Enogastronomía en Santana do Livramento.

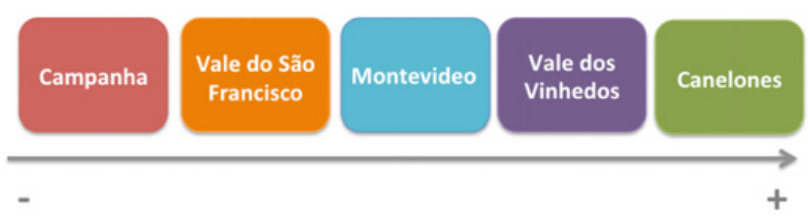

Figura 5. Escala de las regiones con relación al turismo y promoción del vino y de la región. Fuente: los autores.

El turismo en el Valle de San Francisco tiene dos públicos: el del ocio y de los negocios. El turismo de negocios se centra en Petrolina directamente relacionada a la irrigación del polo de las frutas. En términos de placer, el punto turístico principal es el río San Francisco; las agencias locales ofrecen rutas y programas, incluyendo paseos en bote y visitar la presa de Sobradinho. En términos de turismo del vino, las bodegas están proponiendo rutas relacionadas con el río San Francisco, incluyendo otros lugares, como las industrias de frutas, comida o vino. El turismo del vino está creciendo en importancia y se está convirtiendo en una actividad clave para algunas bodegas en términos de marketing, sino también para poner de entrada. Al mismo tiempo, el turismo del vino se está convirtiendo en una atracción para la región; una encuesta a los turistas que visitaron la región Casa Nova mostró que el $57 \%$ de los turistas de ocio fueron motivados por el enoturismo [16].

En Uruguay, el enoturismo y el turismo rural son dos aspectos que se están trabajando y a los cuales se les da una importancia particular. Sin embargo permanece como una situación excepcional ya que sigue siendo la actividad principal la viticultura o la bodega. Cambios en la cultura del consumo del vino hace que se necesite un proceso de reposicionamiento del producto en el mercado local.

Canelones y Montevideo ocupan la región metropolitana, por lo cual muchas de las estrategias de desarrollo están integradas, e incluso, las bodegas integrantes de la Asociación Los Caminos del Vino, son de ambos departamentos. Esta cercanía geográfica, facilita algunos de los procesos de abordaje integral de la propuesta de enoturismo. Un destaque importante ha sido la celebración del "Día de Enoturismo", que tiene lugar en el segundo fin de semana de cada año. Este evento, que se concentra la actividad bodegas, el turismo, la gastronomía y el valor cultural y patrimonial, es único en América Latina y está vinculado a proyectos desarrollados por Red Europea de Ciudades del Vino (RECEVIN). Las actividades se desarrollan en cuatro departamentos (Montevideo, Colonia, Maldonado y Montevideo). Las celebraciones del Día Enoturismo tienen el apoyo del Gobierno Federal (a través del Ministerio de Turismo y INAVI) y los Departamentos.

\section{Considerations}

Los análisis de este artículo han permitido evaluar, de forma exploratoria, las condiciones de competencia presentes en las áreas de la actividad reciente enoturística en ambos países considerados de estar surgiendo en la producción mundial de vino: Brasil y Uruguay. En términos generales, era posible establecer un marco analítico para las dos realidades, que en el futuro será ampliado y profundizado. El turismo del vino, como fue 
explicado claramente, es un sector económico importante en las regiones analizadas, y su papel ha sido cada vez más importante para la comprensión de la dinámica del "negocio del vino" en la actualidad.

La creciente oferta de productos de mayor calidad ha cambiado todas las regiones analizadas. Hasta la década de 1970 se hizo hincapié en la producción de uvas y vinos en territorios más analizados, desde finales de 1980 el vino asumió un papel de liderazgo en la cadena de producción de la uva y el vino en ambos países. Y a partir de finales de la década de 1990, un valor creciente movimiento, el suministro asociado de vino con el turismo local y la gastronomía añadió, ha dado lugar a una nueva forma de relación con sus consumidores cadena: el turismo del vino. Este movimiento, presente tanto entre los pequeños productores y entre las bodegas más grandes, permite el desarrollo de un "turismo/economía de experiencia." Las experiencias son considerados como diferentes ofertas económicas, y diferentes servicios, debido a que el concepto de experiencia presupone una forma de agregar valor en la prestación de servicios.

En gran medida, estas iniciativas relacionadas con el turismo del vino están surgiendo, sobre todo en la región de la Sierra del Río Grande del Sur (Brasil) de los proyectos de las indicaciones geográficas (IG), que comenzó en la región desde la década de 2000. El IG se caracterizan como una posibilidad para facilitar estrategias de inclusión social y económica (Niederle; VITROLLES, 2010), que actúa como refuerzo de los instrumentos de los territorios. Según Farias ... [1], el fortalecimiento de las marcas de la IG despejar una característica cada vez más presente en la industria del vino: la dependencia de los agricultores tienen centros urbanos.

El crecimiento del turismo del vino supone la búsqueda de los turistas que vienen, sobre todo en las zonas urbanas. El clima, la cultura y el imaginario vinculado a la producción de vino ayuda a crear una "atmósfera de vino" en sí. En este sentido, se demuestra el argumento Wanderlei [17], que con respecto a "nuevas ruralidades brasileños", es la dificultad de distinguir a la población rural de las zonas urbanas, con más bien un "continuo rural-urbano". Esta característica se presenta también por otros autores con respecto a los cambios en la vida rural en otros países $[18,19]$. Por lo tanto, las directivas que tengan la intención de fortalecer el turismo del vino, especialmente entre los pequeños productores deben pasar necesariamente por la creación de guiones y la viabilidad de acceso de turistas urbana a las zonas rurales.

Por medio del marco analítico utilizado, fue posible verificar las similitudes y diferencias entre las regiones enoturísticas en ambos países, en particular, los elementos que componen los factores de competitividad e innovación. Se puede observar que las regiones brasileñas acaban teniendo papel importante, ya que atraer a los principales actores institucionales, incluidas las organizaciones educativas y de investigación. Por otro lado, las regiones uruguayas tienen una densidad y estructura importante, lo que puede dar todo su apoyo al desarrollo del turismo del vino, especialmente de manera complementaria a la disposición de turismo existentes en el país. Por lo tanto, se puede decir que el turismo del vino elaborado en Uruguay está más vinculado a la visita de las unidades de producción (bodegas y sus viñedos), mientras que el turismo del vino brasileña está más asociada con la experiencia y la vida al turista con la "atmósfera" de vino involucrando así a un mayor número de empresas e instalaciones turísticas. En parte, este comportamiento turista brasileño es debido al conocimiento incipiente que esto tiene sobre el vino, que puede ser determinada por el consumo per cápita de menos de 2 litros (IBRAVIN).

Es interesante destacar que Uruguay y Brasil ya tienen una trayectoria de aproximación para el enoturismo, a través de acciones tales como el $5^{\circ}$ Congreso Latinoamericano de Enoturismo Montevideo, promovido en el año de 2015 por AENOTUR (Asociación de Turismo del Vino) y ATEU (Asociación de Enoturismo de Uruguay), cuando se discutió la creación de grupos de trabajo y el intercambio de experiencias. Otro ejemplo es el Festival Binacional de Enogastronomía que ocurre en Santana do Livramento y Rivera, una región que también tiene una iniciativa de formación técnica binacional, una colaboración entre el Instituto Federal Sul Riograndense (IFSul) y la Universidad del Trabajo del Uruguay (UTU); la carrera técnica en los alimentos/cocina ocurre en la Escuela Técnica de Rivera. En este sentido, se pueden vislumbrar las perspectivas de cooperación entre Brasil y Uruguay para intensificar, lo que podría generar una sinergía y fortalecer los factores de competitividad en ambos países. El enoturismo puede ser un elemento complementario, lo que permite una ampliación de las relaciones comerciales y la cooperación existentes entre los dos países.

\section{Referencias}

[1] Farias, C. V. S. O papel das instituições na formação e transformação da vitivinicultura da Serra Gaúcha: possibilidades de interpretações do desenvolvimento rural pela nova economia institucional (2016)

[2] Coutinho, Luciano G.; Ferraz, J. C. Estudo da competitividade da indústria brasileira. (Papirus. Editora da Universidade Estadual de Campinas, 1994)

[3] Barbosa, F. V. in Compet. alianças estratégicas e gerência Int. (Rodrigues, S. B.) 21-40 (Atlas, 1999)

[4] Lapolli, J. N. et al. A competitividade da viticultura brasileira: análise setorial e programa de ação com destaque para o Rio Grande do Sul. (Banrisul/EMBRAPA - CNPUV/SEBRAE, 1995)

[5] Ferraz, João Carlos; Kupfer, David; Haguenauer, L. Made in Brazil: desafios competitivos para a indústria. (Campus, 1997)

[6] Porter, M. E. A vantagem competitiva das nações. (Campus, 1993)

[7] Porter, M. E. Cluster and the new economics of competition. Harv. Bus. Rev. 77-99 (1998)

[8] Hall, C. M., Sharples, L., Cambourne, B. \& Macionis, N. Wine tourism around the world: development, management and markets. (Routledge, 2000)

[9] Sommers, B. J. The Geography of Wine: How Landscapes, Cultures, Terroir, and the Weather Make a Good Drop. (Penguin Group US, 2008)

[10] Falcade, I. in Abordagens teórico-metodologicas em Geogr. agrária (Marafon, G. J., Rua, J. \& Ribeiro, M. A.) 225-253 (EdUERJ, 2007)

[11] Falcade, I. \& Medeiros, R. M. V. Transformações no vale dos vinhedos: dinâmicas urbanas no rural? in An. do I Simpósio Nac. o Rural e o Urbano no Bras. (USP) (Edusp, 2006) 
[12] OIV. OIV Vine and Wine Outlook 2010-2011. (OIV, 2014)

[13] Falcade, I. A Paisagem como Representação Espacial: A paisagem Vitícola como Símbolo da Indicação de Procedência de vinhos das regiões Vale dos Vinhedos Pinto Bandeira e Monte Belo do Sul (Brasil) (2011)

[14] IBGE (Instituto Brasileiro de Geografia e Estatística). Sistema IBGE de Recuperação Automática - SIDRA. Banco Dados Agreg. (2015). at <http://www.sidra.ibge.gov.br/>

[15] Flores, S. S. Desenvolvimento territorial sustentável a partir da vitivinicultura: o caso dos 'Vinhos da Campanha' (2011)
[16] Bahia (Brasil). Caracterização do turismo receptivo na Bahia, 2011: Casa Nova. (2012). at <http://observatorio.turismo.ba.gov.br/ pesquisas-de-segmentos/enoturismo/ 2011-2/>

[17] Wanderley, M. N. B. A emergência de uma nova nuralidade nas sociedades modernas avançadas?: o rural como espaço singular e coletivo. Estud. Soc. $e$ Agric. 15, 87-145

[18] Cloke, P. in Handb. Rural Stud. 126-147 (Sage Publications, 2006)

[19] Ploeg, J. D. van der et al. Rural development: from practices and policies towards theory. Sociol. Ruralis 40, 391-408 (2000) 